OPEN ACCESS

Edited by:

Gul Dolen,

Johns Hopkins University, USA

Reviewed by:

Jeffrey Varner,

Cornell University, USA

Don Kulasiri,

Lincoln University, New Zealand

*Correspondence:

Brian J. O'Roak oroak@ohsu.edu

Specialty section:

This article was submitted to Systems Biology,

a section of the journal

Frontiers in Neuroscience

Received: 16 July 2015 Accepted: 26 November 2015 Published: 17 December 2015

Citation: Barnard RA, Pomaville MB and O'Roak BJ (2015) Mutations and Modeling of the Chromatin Remodeler

CHD8 Define an Emerging Autism Etiology. Front. Neurosci. 9:477. doi: 10.3389/fnins.2015.00477

\section{Mutations and Modeling of the Chromatin Remodeler CHD8 Define an Emerging Autism Etiology}

\author{
Rebecca A. Barnard ${ }^{1}$, Matthew B. Pomaville ${ }^{1,2}$ and Brian J. O'Roak ${ }^{1 *}$ \\ ${ }^{1}$ Department of Molecular \& Medical Genetics, Oregon Health \& Science University, Portland, OR, USA, ${ }^{2}$ Department of \\ Biology, California State University, Fresno, CA, USA
}

Autism Spectrum Disorder (ASD) is a common neurodevelopmental disorder with a strong but complex genetic component. Recent family based exome-sequencing strategies have identified recurrent de novo mutations at specific genes, providing strong evidence for ASD risk, but also highlighting the extreme genetic heterogeneity of the disorder. However, disruptions in these genes converge on key molecular pathways early in development. In particular, functional enrichment analyses have found that there is a bias toward genes involved in transcriptional regulation, such as chromatin modifiers. Here we review recent genetic, animal model, co-expression network, and functional genomics studies relating to the high confidence ASD risk gene, $\mathrm{CHD} 8$. CHD8, a chromatin remodeling factor, may serve as a "master regulator" of a common ASD etiology. Individuals with a CHD8 mutation show an ASD subtype that includes similar physical characteristics, such as macrocephaly and prolonged Gl problems including recurrent constipation. Similarly, animal models of CHD8 disruption exhibit enlarged head circumference and reduced gut motility phenotypes. Systems biology approaches suggest $\mathrm{CHD} 8$ and other candidate ASD risk genes are enriched during mid-fetal development, which may represent a critical time window in ASD etiology. Transcription and CHD8 binding site profiles from cell and primary tissue models of early development indicate that $\mathrm{CHD} 8$ may also positively regulate other candidate ASD risk genes through both direct and indirect means. However, continued study is needed to elucidate the mechanism of regulation as well as identify which CHD8 targets are most relevant to ASD risk. Overall, these initial studies suggest the potential for common ASD etiologies and the development of personalized treatments in the future.

Keywords: autism, autism spectrum disorder (ASD), CHD8, systems biology, co-expression networks, functional genomics, subtype, de novo mutations

\section{INTRODUCTION}

Autism spectrum disorder (ASD) is a lifelong neurodevelopmental disorder characterized by restricted, repetitive behaviors and impaired communication and social interactions (American Psychaitric Association, 2013). ASD can have a considerable impact on quality of life as many people with ASD experience difficulties communicating, developing relationships, and managing restrictive behaviors (American Psychaitric Association, 2013). Additionally, the prevalence of ASD appears to have steadily increased over the last few decades, from $<0.5 \%$ of American 
school aged children in the 1970s to $2 \%$ in 2012 (Blumberg et al., 2013). The most recent estimates for the median worldwide prevalence of autism and pervasive developmental disorders is about 1 in 160 children (Elsabbagh et al., 2012) and 1 in 68 children in the US (Centers for Disease Control, 2014). The apparent rise in ASD has prompted a great deal of interest and research into identifying the underlying causes of the disorder.

Many studies have indicated a strong genetic contribution for ASD risk (Geschwind and State, 2015). Early twin and familial studies demonstrated compelling evidence for the heritability of ASD due to the high rate of concordance for ASD between monozygotic twins $(0.62-0.94)$ as opposed to dizygotic twins $(0.05-0.62)$ and increased relative recurrence risk for siblings of affected individuals (10.1\%; Abrahams and Geschwind, 2008; Risch et al., 2014; Colvert et al., 2015). However, the overall ASD genetic architecture is complex with (1) risk being conferred by many independent genomic loci and contributions from common and rare variants, as well as new or de novo mutations, (2) factors that range in size, from single base changes to large chromosomal deletions/duplications or other rearrangements, (3) impacts that include necessary and sufficient single variants, to small (oligogenic) and large (polygenic) sets of factors, (4) the relative contributions of these factors are likely different in subpopulations, such as simplex (single sporadic presentation in an individual, with no previous family history) vs. multiplex (multiple affected individuals in a family), or other demographics yet to be elucidated. These realities have hampered traditional gene discovery methods.

Despite these challenges, recent advances in sequencing technology and novel approaches have begun to unlock the genetics of idiopathic ASD and high confidence risk genes are beginning to emerge that shed new light on the underlying biology of this disorder. Here, we highlight one such gene, Chromodomain helicase binding protein 8 (CHD8). We review the discovery of $C H D 8$ as one of the most mutated genes in simplex ASD, its molecular function, associated ASD subtype, and its potential role as a master regulator of other candidate ASD risk genes. Although CHD8 is still just one of many ASD risk genes, these data point toward at least one converging neuromolecular mechanism in ASD etiology and the potential stratification of ASD into distinct genetic/biologic subtypes. This promises to have major implications for the future as we strive to develop personalized therapies and precision treatments for individuals with ASD.

\section{GENOMIC DISCOVERY REVOLUTION}

Earlier comparative genomic hybridization and genotyping array studies advanced the genomewide discovery of large regions of chromosomal deletions or duplications, termed copy number variants (CNVs), as substantial contributors to the risk of developing neurodevelopmental disorders (Rosenfeld et al., 2010). CNVs typically involve gains or losses of many genes and can show diversity in penetrance and expressivity. While these CNVs play a critical role in the architecture of neurodevelopmental disorders, with few expectations, it has been difficult to get at the underlying locus or loci that are responsible for this risk. The advent of whole-exome (the entire protein coding exons of the genome) sequencing allowed for mutation detection at single-base resolution and was initially applied to Mendelian disorders (Ng et al., 2010). A number of groups began applying these methods to small cohorts of sporadic cases of a variety of conditions, including idiopathic intellectual disability (ID), ASD, and schizophrenia, focusing on parent-child trios or families (Vissers et al., 2010; Girard et al., 2011; O’Roak et al., 2011; Xu et al., 2011). It was hypothesized that such families would likely be enriched for de novo mutations related to the condition, thereby allowing the identification of novel genetic events with major biologic effect in an unbiased genomwide fashion. These initial studies demonstrated the feasibility of obtaining exome data of sufficient quality across the trios ( $>90 \%$ jointly covered) and filtering strategies to identify the $\sim 1$ true de novo event expected per generation (Veltman and Brunner, 2012). Moreover, they appeared to yield a large number of possible candidate genes for these conditions.

For simplex or sporadic ASD, these efforts were greatly expanded in 2012 with four groups publishing the results of hundreds of families independently (Iossifov et al., 2012; Neale et al., 2012; O’Roak et al., 2012b; Sanders et al., 2012). Mutations occurred in genes with very diverse functions and in $>900$ ASD probands (affected individuals) only six genes exhibited recurrent likely loss-of-function (LoF) mutations, defined as nonsense (premature stop codon resulting in gene truncation), canonical splice-site (altered donor or acceptor splice sites leading to improperly spliced mRNA) or frameshifting nucleotide insertion/deletion (indel), suggesting extreme locus heterogeneity. One of the genes identified with two LoF mutations in 209 ASD affected children from the Simons Simplex Collection (SSC) by O'Roak and colleagues was CHD8, an ATP-dependent chromatin-remodeling factor (O'Roak et al., 2012b; Table 1). CHD8 protein was also part of a large proteinprotein interaction network emerging from the LoF and most severe missense mutations. This network was ranked highly for similarity to previously identified ASD risk genes using a network walking approach. Furthermore, the locus specific mutation rate for CHD8 suggests that it was highly unlikely to find two independent mutations at random (in the single study), providing additional evidence for $\mathrm{CHD} 8$ as an ASD risk factor (O'Roak et al., 2012b). Mutation of CHD8 had not been previously implicated with ASD, except in one concurrent study examining balanced chromosomal abnormalities in ASD and other neurodevelopmental disorders (Talkowski et al., 2012). Talkowski and colleagues mapped a balanced translocation involving 3q25.31 and 14q11.1 [46 XX, $t_{(3 ; 14)}(\mathrm{q} 25.31 ; \mathrm{q} 11.2) \mathrm{dn}$ ], which directly disrupted only one gene, CHD 8 at the $14 \mathrm{q} 11.1$ breakpoint. The proband was diagnosed with ASD, ID, and had dysmorphic facial features. Large de novo deletions of 14q11.2 were previously observed in three other subjects that all exhibited developmental delay (DD) and cognitive impairment and similar dysmorphic features including widely spaced eyes, short nose with broad nasal tip, and unusual helical root formation of the ear (Zahir et al., 2007). The presence or absence of ASD was not noted. The overlapping regions lost in these three 
TABLE 1 | De Novo CHD8 Mutations.

\begin{tabular}{|c|c|c|c|c|c|c|c|c|c|c|}
\hline $\begin{array}{l}\text { Chr } 14 \\
\text { Position }\end{array}$ & $\begin{array}{l}\text { Ref }^{\mathrm{a}} \\
\text { Allele }\end{array}$ & Alt ${ }^{a}$ Allele & $\begin{array}{l}\text { Mutation Location } \\
\text { (HGVS) }^{\text {b }}\end{array}$ & $\begin{array}{l}\text { Mutationc } \\
\text { Type }\end{array}$ & Diagnosis $^{d}$ & $\mathrm{VIQ}^{\mathrm{e}}$ & $N_{V I Q}{ }^{f}$ & FSIQg & $\begin{array}{l}\text { Database } \\
\text { /Cohort }^{\mathrm{h}}\end{array}$ & Author, Year \\
\hline $2,189,9618$ & $\mathrm{G}$ & C & p.Ser62X & Ns & ASD & 75 & 78 & 74 & SSC & O'Roak et al., 2012a \\
\hline $21,899,168$ & C & $\mathrm{T}$ & p.Arg212Gln & Ms & ASD & & 72 & & TASC & O'Roak et al., 2014 \\
\hline $21,895,989$ & del(47)† & A & c.1593_1601+38del† & Ssv & ASD & & & & SSC & lossifov et al., 2014 \\
\hline $21,882,516$ & $G$ & $\mathrm{~T}$ & p.Gln696Lys & Ms & ASD & 88 & 125 & & TASC & O'Roak et al., 2014 \\
\hline $21,878,133$ & $\mathrm{G}$ & GT & p.Tyr747X & Fs & ASD & 25 & 38 & 32 & SSC & O'Roak et al., 2012a \\
\hline $21,876,700$ & A & G & p.Leu834Pro & Ms & ASD & & & & ASC & De Rubeis et al., 2014 \\
\hline $21,876,489$ & C & $\mathrm{T}$ & p.Met904lle & Ms & ASD/ID & & 63 & & TASC & O'Roak et al., 2014 \\
\hline $21,871,373$ & $\mathrm{~T}$ & C & c. $3519-2 A>G$ & $\mathrm{Sp}$ & ASD & 37 & 47 & 43 & SSC & O'Roak et al., 2012a \\
\hline $21,871,790$ & C & A & p.Glu1114X & Ns & ASD & 27 & 41 & 34 & APP & Bernier et al., 2014 \\
\hline $21,871,178$ & G & A & p.Gln1238X & Ns & ASD & 20 & 34 & 27 & SSC & O’Roak et al., 2012b \\
\hline $21,870,652$ & C & $\mathrm{T}$ & p.Arg1242Gln & Ms & ASD & & & & ASC & De Rubeis et al., 2014 \\
\hline $21,870,169$ & G & A & p.Arg1337X & Ns & ASD & 85 & 86 & 84 & SSC & O'Roak et al., 2012a \\
\hline $21,868,219$ & G & A & p.Arg1580Trp & Ms & ASD & 97 & 74 & & TASC & O'Roak et al., 2014 \\
\hline $21,867,866$ & $\mathrm{~T}$ & G & p.Tyr1642LeufsX25 & Ssv-Fs & ASD & & & & ASC & De Rubeis et al., 2014 \\
\hline $21,867,866$ & $\mathrm{~T}$ & G & c. $4818-2 A>C$ & Ssv & ASD & & 93 & & TASC & O'Roak et al., 2014 \\
\hline $21,865,980$ & A & $\mathrm{T}$ & p.Ser1606ArgfsX8 & Ssv-Fs & ASD & & & & ASC & De Rubeis et al., 2014 \\
\hline 21,865,980 & $A$ & $\mathrm{~T}$ & $c .5051+2 \mathrm{~T}>\mathrm{A}$ & Ssv & ASD & 96 & 103 & & TASC & O'Roak et al., 2014 \\
\hline $21,862,642$ & C & $\mathrm{T}$ & p.Gly1602ValfsX15 & Ssv-Fs & ASD & & & & ASC & De Rubeis et al., 2014 \\
\hline 21,862,535 & G & A & p.Arg1834X & Ns & ASD & & & & TASC & O'Roak et al., 2014 \\
\hline $21,862,159$ & CC & C & p.Glu1932SerfsX3 & Fs & DD/ID/ASD & & & 46 & Troina & Bernier et al., 2014 \\
\hline $21,861,643$ & TCTTC & $\mathrm{T}$ & p.Glu2103ArgfsX3 & Fs & ASD & 44 & 67 & 59 & SSC & O’Roak et al., 2012a \\
\hline $21,861,376$ & ACT & A & p.Leu2120ProfsX13 & Fs & ASD & 90 & 93 & 91 & SSC & O’Roak et al., 2012b \\
\hline $21,860,919$ & C & A & p.Ser2173X & Ns & ASD/SHZ & & & & & Mccarthy et al., 2014 \\
\hline $21,861,328$ & $\mathrm{~T}$ & $\mathrm{TC}$ & p.Glu2136ArgfsX6 & Fs & ID & & & $<40$ & Troina & Bernier et al., 2014 \\
\hline \multirow[t]{2}{*}{$21,854,022$} & GGGT & $\mathrm{G}$ & p.His2498del & $\mathrm{Aa}$ & ASD & 84 & 98 & 92 & SSC & O'Roak et al., 2012a \\
\hline & & & t.3;14,q25.31;q11.2 & $\operatorname{Tr}$ & ASD/ID & & & & AGRE & Talkowski et al., 2012 \\
\hline
\end{tabular}

a VCF format, hg19 coordinates.

${ }^{b}$ Accession number: NP_001164100.1; Ref Seq number: NM_001170629.1.

${ }^{c}$ Fs, Frameshift; Ns, Nonsense; Tr, Translocation; Aa, Single amino acid deletion; Sp, Splice; Mns, Missense near splice site; Ms, Missense; Ssv, Splice site variant.

${ }^{a} A S D$, Autism Spectrum Disorder; ID, Intellectual Disability; SHZ, Schizophrenia.

eVIQ, Verbal I.Q.

${ }^{f}$ NVIQ, Non Verbal I.Q.

${ }^{g}$ FSIQ, Full Scale I.Q.

hSSC, Simons Simplex Collection; AGRE, Autism Research Genome Exchange; ASC, Autism Sequencing Consortium; TASC, The Autism Simplex Collection.

' CAAGCTCAAGTGAGTACTCCTTGCTACTGTGATGGGACGT.

cases included CHD8 and SUPT16H. CHD8 was suggested as a possible candidate gene for these abnormalities (Zahir et al., 2007).

In an effort to identify additional mutations and firmly implicate CHD8 and other strong candidates, targeted resequencing studies utilizing cost-effective modified molecular inversion probes (MIPs) were applied to larger cohorts of ASD and DD probands. In the first such study, the protein coding regions of 44 genes were successfully sequenced in 2446 ASD probands from the SSC (O'Roak et al., 2012a). Seven additional de novo CHD8 mutations were identified: six LoF variants and a single amino acid deletion, including a mutation missed by exome sequencing (Table 1). This finding was highly significant as determined by a model of recurrent gene mutation, firmly implicating de novo LoF mutations in CHD8 with ASD risk $\left(p<2 \times 10^{-9}\right)$. Including exome data available at the time, 9/2573 (0.35\%)
SSC probands carried a CHD8 mutation, making this locus one of the most frequently mutated in sporadic/simplex ASD.

These resequencing studies were further extended in a broader neurodevelopmental cohort of 3730 children with ASD or DD (Bernier et al., 2014), 898 ASD confirmed probands from The Simplex Autism Collection (TASC; O’Roak et al., 2014), as well as $\sim 2500$ unaffected children from SSC or TASC. These studies identified six additional de novo LoF variants, four de novo missense variants, and one inherited LoF variant. LoF mutations were not seen in unaffected siblings or in an additional 6503 general population control (Bernier et al., 2014; O'Roak et al., 2014). In total, 16 de novo CHD8 mutations (0.46\%, Poisson 95\% CI: $0.26-0.75 \%)$ were identified in the SSC and TASC cohorts, both of which required probands to meet ASD criteria on the Autism Diagnostic Interview-Revised (ADI-R) and Autism Diagnostic Observation Schedule (ADOS; Lord et al., 1994, 
1999). Combined with the more broadly defined Bernier et al. cohort, the observed de novo rate is $0.3 \%$. These findings further strengthen the association of de novo mutations in CHD8 with ASD risk by identifying mutations in independent cohorts.

In late 2014, two large-scale exome sequence studies were also published, including $\sim 2500$ families from the SSC (Iossifov et al., 2014) and 1478 families and 1673 case-only by the Autism Sequencing Consortium (De Rubeis et al., 2014). In the SSC one new additional CHD8 mutation was identified (nine previously identified including MIP and exome data). The ASC identified two CHD8 de novo missense mutations from the family and three splice site variants from case only exome data (through genotyping selected parents; De Rubeis et al., 2014). Risk genes were identified using a statistical approach called TADA (Transmission and De Novo Association), that integrates family-based (de novo and transmitted) and case-control data (He et al., 2013). In this analysis, CHD 8 was also identified as one of the top 13 ASD risk genes with a false discovery rate (FDR) of $<0.01$. In summation, both exome and targeted resequening data have firmly demonstrated that de novo CHD8 mutations play an important role in ASD risk.

\section{CHD8 MUTATIONS DEFINE A SUBTYPE OF ASD}

CHD8 is not only one of the most recurrently mutated genes in sporadic ASD, but also appears to give rise to a distinct ASD phenotype. The first two probands with CHD8 mutations identified from the early sequencing efforts of O'Roak and colleagues, interestingly, had unusually large head circumferences (macrocephaly; O'Roak et al., 2012b). Similarly, the index case from Talkwoski et al. carrying the CHD8 disrupting balanced translocation also presented with macrocephaly and had dysmorphic facial features including prominent forehead and eyes and posteriorly rotated ears (Talkowski et al., 2012). Noting this, head circumference of the the eight probands with LoF mutations in CHD8 identified from targeted re-sequencing of the SSC (O'Roak et al., 2012a) was examined. They found that head size was significantly larger in the individuals with CHD8 mutations (greater than two standard deviations) as opposed to those without. Additionally, about $2 \%$ of SSC probands with macrocephay had CHD 8 mutations. Macrocephaly has been recognized in other genetic etiologies of ASD, mainly loss of PTEN function and deletions in 16p11.2 (Butler et al., 2005; Shinawi et al., 2010).

To expand the group of individuals with known CHD8 mutations and assess the potential for a CHD8 related subphenotype, patients identified through the exome and targeted sequencing $\mathrm{ASD}$ and $\mathrm{DD}$ cohorts were invited to participate in a comprehensive structured evaluation using a battery of standard cognitive, adaptive, and language tests $(n=$ 8; Bernier et al., 2014). Including previous clinical reports on other subjects $(n=7)$, phenotypic data on 15 total patients with disruptive CHD8 variants were included in the evaluation (13 de novo, one inherited, and one of unknown origin; Figure 1A and Table 2). ASD was the most common
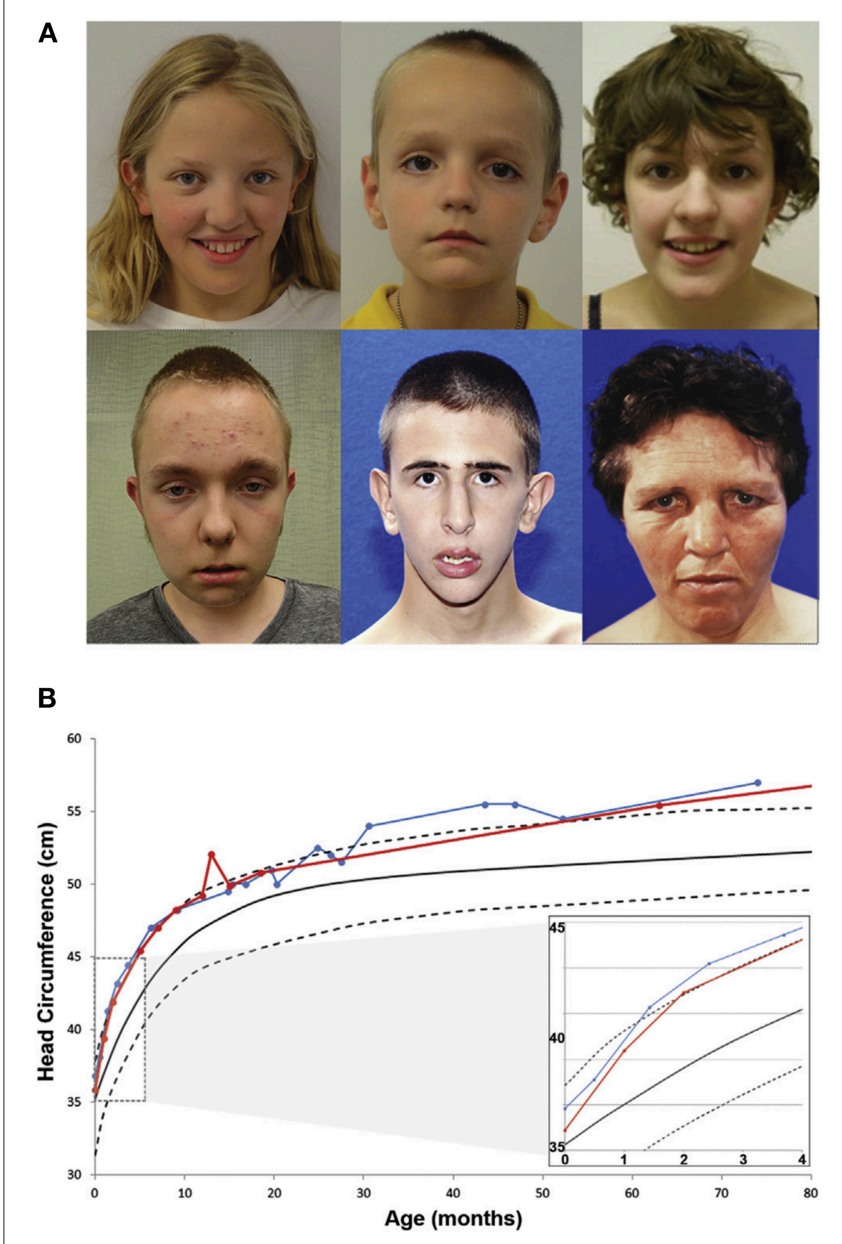

FIGURE 1 | Phenotypic characteristics of patients with $\mathrm{CHD8}$

Mutations. (A) Common facial features of patients with CHD8 mutations include macrocephaly, hypertelorism, down-slanted palpebral fissures, broad nose, pointed chin, and prominent supra-orbital ridge. (B) Longitudinal head circumference data for two patients (red and blue). At 2 months after birth, orbital frontal head growth is pronounced. Head growth continues to be in the 97th percentile throughout childhood. Figure originally published in Bernier et al. (2014) used with permission.

TABLE 2 | Phenotypic Summary of Patients with CHD8 Mutations.

Patient characteristics

Number (\%)

ASD

$13 / 15(87 \%)$

Tall stature

$12 / 14(86 \%)$

Macrocephaly*

$12 / 15$ (80\%)

Gl problems*

$12 / 15$ (80\%)

Sleep problems

$10 / 15(67 \%)$

Intellectual disability

$9 / 15(60 \%)$

*Indicates significantly different from the typical ASD population.

diagnosis with 13 of 15 meeting a strict diagnosis on both "gold standard approaches," ADI-R and ADOS. Macrocephaly, defined as orbitofrontal circumference greater than two standard deviations of age and sex matched means, was exhibited in 12 of 
15 patients (Table 2). Head circumference velocity data $(n=2)$ showed an initial orbital overgrowth within the first 2 months post birth and a continued trajectory of large head growth at or above the 97th percentile throughout early childhood (Figure 1B). The proportion of CHD8 mutation carriers with macrocephaly is significantly greater than that seen in the typical ASD population $\left(p=2.1 \times 10^{-21}\right)$. There were also many similarities in facial features among the group including: prominent forehead, wide set eyes, broad nose with full nasal tip, and pointed chin (Figure 1A). A majority also reported gastrointestinal (GI) problems, including recurrent constipation, and sleep problems, particularly with falling asleep (Table 2). Two patients described suffering from an inability to sleep for two-three straight days. Cognitive impairment was also pervasive, but intelligence spanned a wide range with some in normal range. Moreover, there is indication that additional symptoms may manifest as the children age. Three of the three female patients (including the translocation case) experienced precocious puberty (Talkowski et al., 2012; Bernier et al., 2014).

The implications of a CHD8 specific phenotype suggest ASD could be stratified into different subtypes based on genetic etiology. Identification of these subtypes would provide insight into ASD presentation and pathology in genetically homogenous populations and aid in the development and evaluation of treatment approaches (Bernier et al., 2014). Other high confidence ASD risk genes that exhibit recurrent loss of function mutations, such as ADNP and DYRK1A also appear to be associated with emerging subphenotypes (Helsmoortel et al., 2014; Bronicki et al., 2015; Ji et al., 2015). CHD8 appears to have a great deal of specificity for the ASD phenotype as almost all individuals identified from the screen met the criteria for ASD even though the cohort was not strictly defined for ASD individuals. This however may often not be the case as other strong ASD risk genes show more variable expressivity. For example, SYNGAP1 and CHD2 have been independently implicated in other neurological disorders such as epileptic encephalopathies (Carvill et al., 2013). Future studies examining how these similar genetic mutations span our current diagnostic and clinical boundaries may provide novel insights into the development of common neuropathologies.

\section{A CHROMATIN MODIFIER WITH DIVERSE FUNCTIONS}

CHD8, previously called Duplin, was first identified in a screen for novel interactors within the canonical Wnt/ $\beta$-catenin pathway using a yeast two-hybrid assay with a cDNA library generated from rat brain (Sakamoto et al., 2000). CHD8 is a member of the chromodomain-helicase-DNA binding protein family. The CHD family is characterized by a SNF-2 like ATPase and two chromo (chromatin organization modifier) domains (Marfella and Imbalzano, 2007). The first CHD gene, CHD1, was identified in a search for Ig promoter binding proteins (Delmas et al., 1993; Woodage et al., 1997) At least nine other members have been characterized to date with varying patterns of expression in timing and localization. The CHD family is a structurally diverse group comprised of three main subfamilies categorized by the presence of certain functional domains (Marfella and Imbalzano, 2007). CHD1 and CHD2 proteins contain DNA binding domains near the C-terminus that are thought to recognize AT rich motifs. CHD3 and CHD4 lack DNA binding domains and instead have PHD (plant homeo domain) Zinc finger like domains that may recognize methylated histones and potentially have repressive functions. CHD5-CHD9 also have DNA binding domains, along with additional functional domains located at the C-terminus. $\mathrm{CHD} 8$, as well as $\mathrm{CHD} 7$ and CHD9, contain a BRK domain, which is often seen in other SWI/SNF like proteins, and a DNA binding domain. Other SWI/SNF like complexes, such as BAF, regulate neural tube closure and neuron progenitor identity and differentiation (Ronan et al., 2013). Based on its ATP-dependent chromatin remodeling capabilities and SWI/SNF like domains, a potential role for CHD8 may lie in the regulation of neuronal progenitor cells.

Studies of CHD8 protein in the context of human cellular function show that it is involved in the regulation of transcription factor activity. CHD8 is able to activate the transcription of genes driven by U6 promoters through interactions with the transcription factor hStaf (Yuan et al., 2007). CHD8 is also required for $\mathrm{CTCF}$, a major transcriptional repressor, to function as an inhibitor and insulator (Ishihara et al., 2006). CHD8 can also regulate RNA synthesis as well due to interactions with RNA polymerase II (Rodríguez-Paredes et al., 2009). CHD8 also promotes cellular proliferation in multiple human-derived cell types. Cells with CHD8 knockdown were found to have highly reduced S-phase cell populations and increased populations of cells arresting in G1 (Rodríguez-Paredes et al., 2009). This effect was later found to be a result of CHD8 binding to Sphase-dependent promoters and recruiting E2F, a transcription factor which controls cell cycle regulation (Subtil-Rodríguez et al., 2014). CHD8's role in cell cycle regulation could be a contributing factor in the observed macrocephaly exhibited by individuals with CHD8 LoF mutations. While macrocephaly may suggest overproliferation, in mouse models deficient in PTEN, macrocephaly appears to be attributed to increased cell body or soma size. Similarly, if cells are unable to progress through the cell cycle due to CHD8 loss, they may continue to grow in size (Kwon et al., 2001; Luikart et al., 2011). However, both CHD8 upregulation and downregulation has been observed in cancers suggesting CHD8 cell cycle regulation is likely complex and further study will be needed to determine how it impacts neurodevelopment (Lawrence et al., 2014; Subtil-Rodríguez et al., 2014).

What is also still unclear is the mechanism by which CHD8 controls transcription factor activity. Many studies have demonstrated that CHD8 has affinity for histone $\mathrm{H} 3$ lysine 4 (H3K4me3), a marker for active transcription, and is present at active promoters (Yuan et al., 2007; RodríguezParedes et al., 2009; Sugathan et al., 2014; Cotney et al., 2015). Additionally the loss of CHD8 protein was associated with $\mathrm{CpG}$ hypermethylation and histone hypoacetylation near CTCF binding sites. Though CHD8 did not affect the ability of CTCF to bind DNA, potentially the CTCF-CHD8 complex may 
interact with methyltransferases and histone acetyltransferases to influence the state of nearby chromatin (Ishihara et al., 2006). Thus, CHD8 could promote transcription factor binding by making chromatin more accessible or acting as a scaffold between $\mathrm{H} 3 \mathrm{~K} 4 \mathrm{me} 3$ labeled histones and the transcription factor.

\section{CHD8 DISRUPTION IN ANIMAL MODELS MIMICS HUMAN PHENOTYPES}

In the in vivo setting, CHD8 was first examined in the context of $\beta$-catenin signaling due to its nuclear localization and ability to repress $\beta$-catenin dependent transcription (Sakamoto et al., 2000). In zebrafish, $\beta$-catenin is necessary for axis formation. Dorsal injections of Chd8 mRNA, causing overexpression of the protein, resulted in loss of the head. CHD8 expression in the zebrafish is enriched in the brain and spinal cord at 1 day post fertilization, and becomes progressively restricted to head and gut after 3-4 days post fertilization. Expression appears strongest in the mid-gastrula stage suggesting an important role for $\mathrm{CHD} 8$ in embryonic development regulating $\mathrm{Wnt} / \beta$-catenin activity (Sakamoto et al., 2000).

The association of macrocephaly with a reduction in Chd8 expression is also observed in zebrafish. An increase in the interorbital distance, a surrogate measurement for head circumference, is seen in fish treated with Chd8 targeting morpholinos (Bernier et al., 2014; Sugathan et al., 2014). The distance increases in a morpholino dose dependent fashion. The reduction in CHD8 also coincides with increases in chordin, required in forebrain development, otx2, early marker of midbrain/forebrain neural progenitor cells, and $\mathrm{HuC} / \mathrm{D}$, marker for newborn neurons, indicating elevated proliferation, particularly in the mid and forebrain (Bernier et al., 2014). The zebrafish studies also suggest a role for CHD8 in GI development. Many of the human patients with CHD8 mutations suffer from severe GI issues. Morpholino treated zebrafish have decreased gut motility as evidenced by delayed progression of fluorescent microspheres (Bernier et al., 2014). There is also an overall reduction in enteric neurons in the hindgut.

Similarly in mice, Chd8 expression is at its highest embryonically at E8.5-E12.5 but reduced by E16.5 and low in newborns. Therefore, function is likely restricted to earlymiddle stage embryonic development (Nishiyama et al., 2004). Expression of Chd8 is most prominent in the brain, face, branchial arches, limb buds, and tail. Complete knockout of Chd8 is embryonic lethal. Embryos are resorbed by E9.5 but still recoverable by E8.5. Growth begins to delay as soon as E5.5 and is completely arrested at E6.5 with pronounced apoptosis occurring at E7.5. Heterozygotes though did not have gross abnormalities and were fertile. Interestingly, $\mathrm{Wnt} / \beta$-catenin targets are not increased suggesting that aberrant signaling is likely not the cause for lethality. Rather, Chd8 protein regulates p53 activity by recruiting histone $\mathrm{H} 1$, forming a $\mathrm{p} 53-\mathrm{Chd} 8-\mathrm{H} 1$ complex that represses p53 target gene expression (Nishiyama et al., 2009). Unrestrained p53 activity appears to be the cause for the massive apoptosis seen in embryos. Deletion of p53 in $C h d 8^{-/-}$mice delayed arrest to E10.5 at which time embryos died from a heart defect. Chd8 $8^{-/-}$p53 $3^{+/-}$mice did not recover as well. Taken together, these studies demonstrate a conserved role for CHD8 as a regulator of early formative developmental pathways.

\section{A CHD8-ASD DEVELOPMENTAL WINDOW}

With the great functional diversity observed in the de novo, rare variant, and other candidate ASD risk genes, it seems difficult to reconcile their role in an overarching mechanism for ASD biology. However, a number of biologic network approaches suggest that there may be a converging biology at particular developmental windows and brain regions, which includes CHD8 as a central player. These approaches are leveraging new spatial and temporal genomics data from developing human and nonhuman primates, such as the BrainSpan Atlas, a freely available resource that includes gene expression (microarray and RNAseq) and in-situ hybridization data sets (Shen et al., 2012). As in other organisms, CHD8 expression seems to be most significant early in human development. While CHD 8 is widely expressed in the adult brain, expression is highest at 9-16 post conception weeks (PCW) in both progenitor and post mitotic neocortical layers and then gradually declines (Bernier et al., 2014).

To discover time points of development and brain regions for which candidate and high-confidence ASD risk genes, such as $C H D 8$, may converge, two recent concurrent studies made use of BrainSpan's gene expression data to develop coexpression networks or modules representing genes that share similar expression patterns during development (Parikshak et al., 2013; Willsey et al., 2013). Each of these networks or modules corresponded to different brain regions along progressing stages of development. Remarkably, while the groups took different approaches to develop these networks and assess the potential for enrichment of candidate ASD risk genes they arrived at similar results implicating the mid-fetal time periods as a possible critical window for ASD etiology.

Parikshak and colleagues used BrainSpan RNA-seq data from human neocortex $8 \mathrm{PCW}$ to 12 months after birth to construct co-expression modules by Weighted Gene Coexpression Network Analysis (WGCNA), an unbiased, genomewide method for constructing networks based on pairwise correlations of gene expression (Langfelder and Horvath, 2008; Parikshak et al., 2013).

They identified 17 modules and mapped sets of candidate ASD risk genes onto proteins within the modules. These gene sets included: "asdM12" which was derived from a WGCNA expression module from ASD patient postmortem cerebral cortex and "SFARI ASD" which was a subset of 155 genes from the larger AutDB gene list filtered by gene evidence score (Voineagu et al., 2011; see Table 3 for detailed gene list descriptions). De novo variants identified from 2012 whole-exome sequencing studies performed by O'Roak et al., Sanders et al., and Neale et al. were also mapped onto networks. They then assessed enrichment for candidate ASD risk gene within specific co-expression modules, cortical layers and cell types. The de novo mutation candidate ASD risk genes were more concentrated in modules designated M2 $(p=0.006)$ and M3 $(p=0.0011)$. M2 peaks at $12-22$ PCW and M3 is highly upregulated until PCW 12. The overall set 
TABLE 3 | Referenced Candidate ASD Risk Gene Lists.

\begin{tabular}{|c|c|c|c|c|}
\hline Gene list name & Compilation method & Type & $\begin{array}{l}\text { Number } \\
\text { of genes }\end{array}$ & Author, Year \\
\hline AutDB & $\begin{array}{l}\text { "PubMed" database search for "gene" + "autism" or "autistic" in the titles and } \\
\text { abstracts. Genes are divided into genetic subcategories and assigned evidence } \\
\text { scores. Licensed to SFARI as SFARI Gene by MindSPec. }\end{array}$ & Broad & 667 & Basu et al., 2009 \\
\hline asdM12 & $\begin{array}{l}\text { Set of genes within a co-expression module ("M12") highly correlated with ASD } \\
\text { status. Module was constructed using WGCNA on gene expression from ASD } \\
\text { (19) and control (17) postmortem cortex tissue. Samples from Autism Tissue } \\
\text { Bank and Harvard Brain Bank. }\end{array}$ & Co-expression & 88 & Voineagu et al., 2011 \\
\hline SFARI ASD & $\begin{array}{l}\text { Subset of genes from AutDB filtered for gene category syndromic (S) (associated } \\
\text { with syndromes in which a significant percentage of individuals develop autistic } \\
\text { symptoms) and evidence score 1-4 (high confidence-minimal evidence). }\end{array}$ & Broad & 155 & Parikshak et al., 2013 \\
\hline Willsey set & $\begin{array}{l}\text { Genes identified with de novo LoF mutations from whole-exome sequencing } \\
\text { studies (lossifov et al., 2012; Kong et al., 2012; Neale et al., 2012; O'Roak et al., } \\
\text { 2012a) and an additional } 56 \text { quartets from the SSC. Genes are grouped as, high } \\
\text { confident (hcASD) genes ( } 2 \text { or more de novo LoF mutations), or probable (pASD) } \\
\text { genes (one de novo LoF mutation). The genes in the pASD group were estimated } \\
\text { to have a >50\% chance of being "true ASD" genes based on TADA analysis. }\end{array}$ & Simplex dnLoF & 131 & Willsey et al., 2013 \\
\hline Liu set & $\begin{array}{l}\text { Genes identified by the DAWN algorithm as being implicated in ASD risk using } \\
\text { data from the PFC-MSC co-expression module developed in Willsey et al. and } \\
\text { from whole-exome sequencing studies in family trios (lossifov et al., 2012; Kong } \\
\text { et al., 2012; Neale et al., 2012; O'Roak et al., 2012a; Sanders et al., 2012), } \\
\text { quartets (Willsey et al., 2013) and case-controls (ARRA Autism Sequencing } \\
\text { Consortium, De Rubeis et al., 2014) }\end{array}$ & $\begin{array}{l}\text { dnLoF } \\
\text { Rare transmitted } \\
\text { variants } \\
\text { Co-expression }\end{array}$ & 127 & Liu et al., 2014 \\
\hline
\end{tabular}

of genes in these modules, including non-ASD risk genes, were highly enriched for chromatin modifiers, DNA binding proteins, and transcriptional regulators $\left(p<1 \times 10^{-4}\right)$. Modules M2 and M3 seemed specific to the superficial cortical layers L2-L4 and cells expressing glutamatergic neurons.

In contrast, the "SFARI ASD" and "asdM12" gene sets were enriched in modules M13 (SFARI ASD, $p=0.059$, asdM12, $p=3 \times 10^{-15}$ ), M16 (SFARI ASD, $p=0.0024$, asdM12, $p=3.5 \times 10^{-15}$ ), and M17 (SFARI ASD, $p=0.033$, asdM12, $\left.p=1.0 \times 10^{-7}\right)$. The overall set of genes in these modules had a concentration of synaptic proteins $(p<1 \times$ $10^{-4}$ ) and peaks late in fetal development, starting at $16 \mathrm{PCW}$ and into birth. An enrichment for fragile-X mental retardation protein (FMRP) targets was seen in the M2 module, suggesting a common mechanism between ASD and fragile-X syndrome, in line with the enrichment of FMRP targets first observed by Iossifov et al. (2012). On the other hand, 401 genes implicated in monogenic forms of ID compiled from four publications, showed little enrichment for any one module suggesting the mid-fetal time period may be specific to ASD.

Willsey and colleagues took an alternative approach by focusing on nine high-confidence ASD risk genes, defined as having two or more LoF mutations, and determining their coexpression networks (Willsey et al., 2013; Table 3). These nine genes were used as seeds to generate networks composed of the top positively correlated genes. Gene expression data was also derived from BrainSpan, but based on the Affymetrix GeneChip Human Exon 1.0 ST Array rather than RNA-seq. In addition, all brain regions were considered and 15 periods of development, 5.7 PCW-82 years were examined. Developmental periods were condensed into three time windows resulting in 52 networks based on time and brain region with transcriptional similarity as determined by hierarchical clustering. To see if additional candidate ASD risk genes were enriched in these networks, a list of 122 candidate ASD genes (termed "Willsey set") with at least one LoF mutation identified from whole-exome sequencing studies was generated and mapped onto the networks (Table 3). Permutation testing of enriched networks was used to correct for gene size and GC content. They also looked for enrichment of this gene set within specific cortical layers and cell types. Immunostaining and in-situ hybridization was performed on frontal cortex sections to determine protein expression for five of the high-confidence ASD genes. Similar to Parikshak et al., they found enrichment in networks exhibiting peak expression between 10-19 ( $p=0.003)$ and 13-24 ( $p=0.05)$ PCW (midfetal time period). These networks were specific to the prefrontal and primary motor-somatosensory cortex, but in contrast to Parikshak et al., the networks were specific to the deep layers, L56. Immunostaining revealed that CHD8 and other ASD genes like $S C N 2 A, D Y R K 1 A$, and TBR1 were highly expressed in cortical projection neurons, particularly glutamatergic cell types.

In summary, both groups found a convergence of candidate ASD genes with de novo mutations within the mid-fetal stage of development, 10-24 PCW. The resulting networks appeared specific for glutamaterigic cortical projection neurons, but differed in their implication of specific cortical layers. The cortical projection neurons form synaptic connections early in development and may then be highly sensitive to changes in gene regulation (Willsey et al., 2013). Therefore, de novo mutations in genes like CHD8 may largely impact the midfetal stage of development particularly prefrontal and motorsensory cortex development. 


\section{CHD8 DISRUPTION ALTERS MANY TARGETS INCLUDING OTHER CANDIDATE ASD RISK GENES}

The heterozygous LoF CHD8 mutations seen in ASD likely result in insufficient levels of CHD8 protein and the disregulation of CHD8 targets. The regulatory landscape and widespread reach of CHD8 regulation has been examined in three recent studies using genomic approaches and a number of different neural cellular models that attempt to mimic early development (Figure 2A). Sugathan and colleagues performed knockdown of CHD8 in induced pluripotent stem cell (iPSC) derived neuron progenitor cells (NPCs) using lentiviral delivery of six independent shRNA achieving a range of 38-69\% reduction in CHD8 mRNA expression (Sugathan et al., 2014). They then assessed changes in gene expression using RNA-seq in these knockdown lines and also performed chromatin immunoprecipitation-sequencing (ChIP-seq) in control NPCs to identify CHD8 binding sites. By contrast, Cotney and colleagues performed ChIP-seq to locate CHD8 binding sites (under non-perturbed conditions) in three different systems: human midfetal cortical tissue 1619 PCW, H9 derived human neural stem cells (hNSCs), and embryonic mouse cortex (Cotney et al., 2015). Using primary tissue allowed for representation of mid-fetal stages critical in CHD8-ASD development and to evaluate potential differences between CHD8 binding sites identified in in vivo tissue and in vitro cells. They also performed CHD8 knockdown in hNSCs using two lentiviral shRNA, followed with RNA-seq, to assess changes in gene expression. Finally in the third study, Wilkinson and colleagues performed knockdown of CHD8, achieving about a 50\% reduction, using siRNA in human SK$\mathrm{N}-\mathrm{SH}$ neural progenitor cells followed by RNA-seq (Wilkinson et al., 2015). These studies reveal a complex role for CHD8 in ASD development as it appears to regulate expression of additional candidate ASD risk genes by both indirect and direct means.

Sugathan and colleagues found that knockdown of CHD8 lead to differential expression of some 1756 genes (at nominal $P<0.05 ; 369$ genes at $q<0.05$ Benhamini-Hochberg), most of which were upregulated $(n=1140, p<0.05 ; 286, q<0.05$; Sugathan et al., 2014). The set of down regulated genes $(n=616)$ were enriched for roles in neuronal development. Intriguingly, this same set of downregulated genes was also enriched for candidate ASD risk genes from a large set of 628 genes from AutDB ( $p=3.25 \times 10^{-8}$; Table 3). Though no enrichment for any candidate ASD risk gene sets were observed in the set of up-regulated genes, cancer associated genes, defined by The Cancer Genome Atlas, were enriched in this group. Loss of CHD8 function has been observed in a broad range of tumor types and other chromatin regulators have been implicated in cancer development (Lawrence et al., 2014). This suggests CHD8 may have other important roles extending past development and may be a point of commonality between some cancers and ASD.

In the ChIP-seq data, 7324 reproducible binding sites were identified (Benjamini-Hochberg $q<0.05$ ). CHD8 preferentially bound to regions that in ES cell-derived neuroprogenitor lines from the Roadmap Epigenomics are marked with histone H3 trimethyl Lysine4 (H3K4me3), an indicator of active TSSs (Bernstein et al., 2010). These TSSs were strongly enriched in the CHD8 set, $83 \%$, relative to the genome background, $1 \%$. A small number of binding sites, $4 \%$, overlap enhancer marked sites (H3K4me1), representing a two-fold enrichment. Sequence motifs at CHD8 binding sites were enriched for CTCF and YY1 transcription factor motifs. Genes with CHD8 sites were enriched for $\mathrm{p} 53$, hedgehog, and cell cycle pathways.

Combining the differentially expressed (DE) set of genes with CHD8 binding data, Sugathan and colleagues further explored a number of enrichments using functional classifications, candidate ASD risk gene sets, and other disease gene sets. Overall, only $9.2 \%$ of genes with CHD8 binding sites were $\mathrm{DE}$ in the nominal $\mathrm{P}$ gene set $(29.7 \%$ of all the DE genes). Candidate ASD risk genes from AutDB were enriched in the set of nominally downregulated genes, but not targeted by CHD8 $\left(p=1.09 \times 10^{-9}\right)$. This set of candidate ASD risk genes was enriched for pathways involved in neurodevelopment such as axon guidance and neurotransmitter regulation. Enrichment of the more restricted set of AutDB genes from Parikshak et al., "SFARI ASD" was also observed in the downregulated, nonCHD8 bound genes $\left(p=2.26 \times 10^{-2}\right)$. However, genes from Willsey et al., "Willsey set" were enriched as CHD8 bound genes $\left(p=4.34 \times 10^{-3}\right)$, but were not associated with any changes in gene expression. CHD8 bound genes in the "Willsey set" were enriched for chromatin and transcription. Though these data sets are not independent, they do have different gene representations, with the "Willsey set" more biased toward rare de novo variants. CHD8 binding sites with or without differential expression were explored in the modules described in Parikshak et al. The downregulated genes with no CHD8 binding site were enriched in modules M13, M16, and M17 which correspond to mid-late fetal develop whereas CHD8 bound genes were enriched in modules M2 and M3 corresponding to early-midfetal development. CHD8 bound genes were also highly enriched in TGCA cancer associated genes and also showed no specific enrichment in either up or downregulated genes. No other disease gene sets from the 184 lists for complex disease and traits available from the National Human Genome Research Institute were as significantly enriched as the ASD or cancer gene sets for CHD8 targets or genes affected by CHD 8 knockdown. These data suggest both direct and indirect roles of CHD8 in gene regulation, particularly in the development of ASD and cancer.

Cotney et al. found 9414 reproducible CHD8 binding sites in hNSCs and 4428 in human midfetal brain. Between these two human sets, 2777 sites were overlapping. Chromatin state of hNSC CHD8 binding sites was determined from data generated on hNSCs from their own lab and the ENCODE/Roadmap Epigenomics (Bernstein et al., 2010). Similarly to Sugathan and colleagues, they found that CHD8 had strong affinity for TSSs labeled with $\mathrm{H} 3 \mathrm{~K} 4 \mathrm{me} 3$ or $\mathrm{H} 3 \mathrm{~K} 27 \mathrm{ac}$ and $99 \%$ of promoters bound by CHD8, 8056, were active (Cotney et al., 2015). Additionally, they observed that a small fraction of CHD8 binding sites, 1028, were indicative of enhancer functions. Moreover, CHD8 binding negatively correlated with $\mathrm{H} 3 \mathrm{~K} 27 \mathrm{me}$, indicative of repression. Enriched sequence motifs for transcription factors included CTCF, E2F, YY1, and Sp/Kruppel-like family. 
A

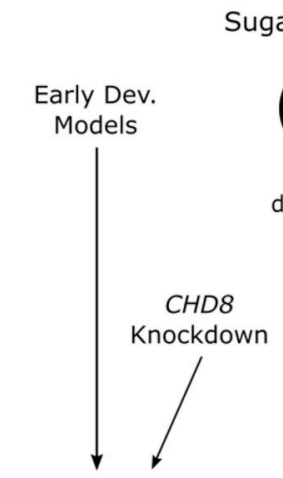

Functional

Genomics

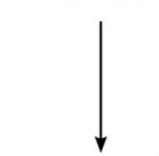

Integrated

Data Analysis
Sugathan, et al.

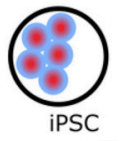

derived NPCs $\downarrow$



Binding

Sites



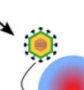

Lentivirus

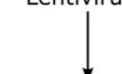

шше

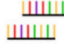

RNA-Seq<smiles>C1CCCCC1</smiles>

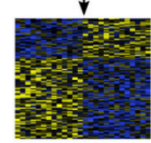

Differential Expression
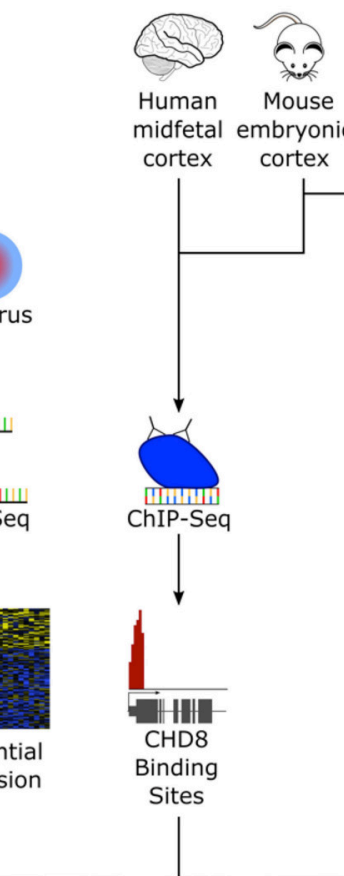

midfetal embryonic

cortex cortex

hNSCS
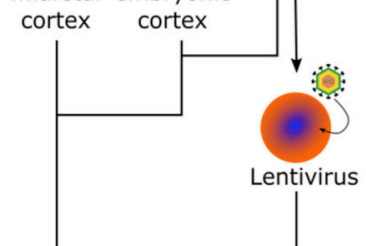

Wilkinson, et al.

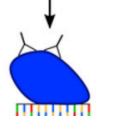

ChIP-Seq

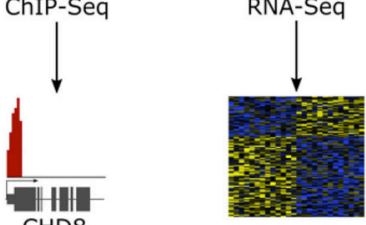

CHD8

Binding

Differential

Expression
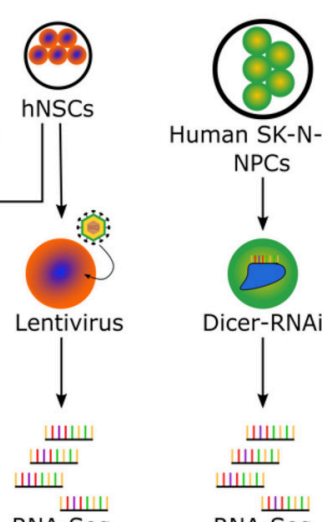

Human SK-N-SH

NPCS

Sites
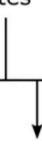

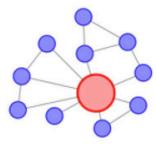

Coexpression

Network 1

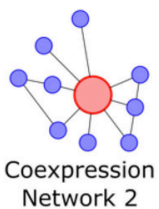

Network 2

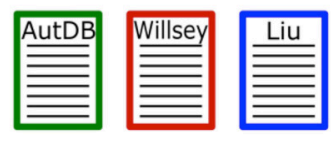

Candidate ASD Risk Gene Sets

B

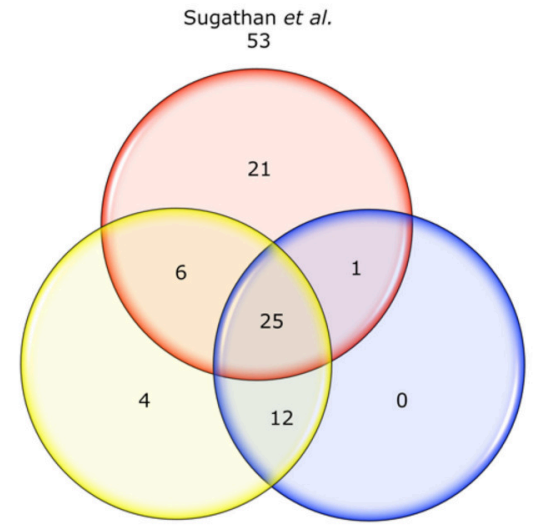

C

$\begin{array}{lll}\text { ADNP } & \text { MYH10 } & \text { SMARCC2 } \\ \text { ARID1B } & \text { NCKAP1 } & \text { SUV42OH1 } \\ \text { ASH1L } & \text { NFKBIL1 } & \text { TRIP12 } \\ \text { BRWD1 } & \text { NUAK1 } & \text { TROVE2 } \\ \text { CNOT3 } & \text { PHF2 } & \text { USP15 } \\ \text { DIP2C } & \text { POGZ } & \text { VCP } \\ \text { FARP1 } & \text { PPP1R15B } & \text { WDFY3 } \\ \text { MED13L } & \text { SETBP1 } & \\ \text { MLL5 } & \text { SETD2 } & \end{array}$

Human Only Cotney et al.

Human + Mouse Conserved Cotney et al.
38

FIGURE 2 | CHD8 functional genomics studies. (A) Flow chart describing functional genomics studies, Sugathan et al., Cotney et al., and Wilkinson et al. including CHD8 knockdown in cellular models of early neural development and ChIP-seq. Cotney et al. also incorporated primary fetal human cortical and embryonic mouse brain tissue into the ChIP-seq analysis. Integrating the gene expression and CHD8 binding profiles, networks of CHD8 regulated genes were constructed and analyzed for enrichment of candidate ASD risk genes. (B) CHD8 targeted candidate ASD risk genes found in the "Willsey set" observed between studies. Genes found in Cotney et al. specifically have CHD8 bound promoters. Human sites in Cotney et al. are shared between hNSCs and brain tissue. Though not included as part of the "Willsey set" in Cotney et al., POGZ is included here. POGZ was noted as having a CHD8 bound promoter and it is one of the high confidence "Willsey set" genes. (C) List of the shared candidate ASD risk genes from the "Willsey Set" bound by CHD8 between both studies and conserved between human and mouse. 
Genes that had CHD8 binding sites identified in both midfetal brain and hNSCs were highly enriched for candidate ASD risk genes from two gene sets as defined by and Liu et al., "Liu set" $(p<0.0001)$ and the "Willsey set" $(p<0.0001$; Willsey et al., 2013; Liu et al., 2014), while genes with binding sites only in hNSCs were not enriched. Candidate ASD risk genes with CHD8 binding sites were enriched for chromatin regulation and modification. CHD8 targets supported by both human datasets were largely evolutionarily conserved. Among mouse (E17.5 cortex), human midfetal brain, and hNSC, 1910 CHD8 binding sites were shared and also enriched for candidate ASD risk genes from both aforementioned gene sets $(p<0.0001)$, further suggesting a conserved mechanism for CHD8 regulation in development. Of the candidate ASD risk genes from the "Willsey set" found to contain CHD8 binding sites, 25 were shared between Cotney et al. and Sugathan et al. and were also conserved in mouse (Figures 2B,C). Enrichment of CHD8 bound proteins was also observed in networks reconstructed from the Willsey et al. 10-19 and 13-24 PCW networks using genes with active promoters (Willsey et al., 2013).

In their knockdown of CHD8 in hNSC, genes with conserved CHD8 binding sites had the greatest fraction of DE genes (56\%) as compared to genes with binding sites only found in hNSC (46\%) or hNSC and human brain (54\%). Cell cycle, Hippo, and p53 pathways were among the pathways most affected. Candidate ASD risk genes actually exhibited the most significant dysregulation compared to any other set of CHD8 targeted genes ( $\sim 60 \%$ for both gene sets). Additionally, those candidate ASD risk genes most DE, tended to be downregulated. While Sugathan et al. did find that candidate ASD risk genes from the Willsey set were enriched as CHD8 targets, they were not significantly $\mathrm{DE}$ in either direction. In contrast, Cotney et al.'s data suggest that CHD8 directly activates this same set of candidate ASD risk genes.

In line with the other studies, Wilkinson et al. found CHD8 reduction had a global impact on gene expression. Interestingly, the top DE genes in this study were noncoding RNAs, the majority of which were upregulated. Noncoding RNAs have only recently been appreciated as having a potential role in neurodevelopment and may be yet another layer in transcriptional control. Enrichment of candidate ASD risk genes Parikshak et al. "SFARI ASD" was only seen in the set of downregulated genes, similar to Cotney et al. as were processes involved in regulation of neuron projection, differentiation and neurogenesis (Parikshak et al., 2013; Wilkinson et al., 2015).

In summation, $C H D 8$ appears to act as a master regulator in the foundational pathways of the developing brain, particularly those that may also be implicated in ASD development. Yet the mechanism for regulation remains unclear. Many of the candidate ASD risk genes are direct targets of CHD8 protein and are also involved in chromatin regulation (Figure 3A). Cotney et al. and Wilkinson et al. indicate that CHD8 directly activates expression of these genes but Sugathan et al. suggest that the presence or absence of CHD8 may not influence expression, despite the genes having a binding site. Other candidate ASD risk genes lack a
CHD8 binding site, but still appear to be affected by CHD8 expression indicating an indirect mechanism of regulation. How CHD8 may be able to influence gene activation or transcription factor activity without directly binding to DNA is not clear, but may involve binding to other co-regulators or chromatin marks (Figure 3B). Continued study will be needed to fully elucidate the mechanism for CHD8 regulation in ASD.

\section{CONCLUSIONS AND FUTURE DIRECTIONS}

From exome sequencing studies, a number of high-confident ASD risk genes have been identified with CHD8 emerging as particularly strong. Both human case studies and animal models suggest a common phenotype for $\mathrm{CHD} 8$ mutation including a significant association with ASD diagnosis and behavior, macrocephaly, and dysfunction in the enteric nervous system. Though ASD is highly heterogeneous, CHD8 systems biology and functional genomics studies give some hint as to the complex nature of the disorder. The CHD8 protein and those of many of the other candidate genes serve as master regulators, influencing the expression of a large network of genes and pathways that control neuron formation, proliferation, and differentiation. The timing of proper neural development is a highly regulated phenomenon, requiring a concerted effort by many different genes. While it's clear that CHD8 controls expression of genes involved in these developmental processes, the manner in which CHD8 regulates them is still unclear. Initial studies suggest that CHD8 may act as a direct repressor by modifying chromatin to make it less accessible. However, the loss of function studies point toward a role for CHD8 as an activator in the emerging ASD gene network. One of the consistent findings from these and previous studies is that CHD8 is associated with actively transcribed genes and has affinity for H3K4me3 labeled promoters suggesting an influential role in global gene expression. CHD8 may function to make promoters more accessible through chromatin remodeling, promote transcription factor binding, or indirectly enhance expression through as of yet, unknown mechanisms that may involve interactions with $\mathrm{H} 3 \mathrm{~K} 4 \mathrm{me} 3$ itself. The mechanism of CHD8 regulation is likely to be gene dependent as well.

It's also still uncertain which CHD8 targets may be relevant for ASD. Though the studies indicate a number of candidate ASD risk genes contain CHD8 binding sites, further functional studies will be required to demonstrate if these genes interact in a physiologic setting. Additionally, although enrichment for candidate ASD risk genes may be seen in specific neuron types, it is still unclear how the alterations in these neurons ultimately relate to behavioral phenotypes. It will be important to determine how CHD8 affects neural circuitry and connect these affects to autistic associated behaviors. Lastly, development of co-expression modules have proved useful in identifying relevant ASD developmental time periods and interactions between candidate ASD risk genes, including CHD8. However, developing methods that integrate multiple types of data such 

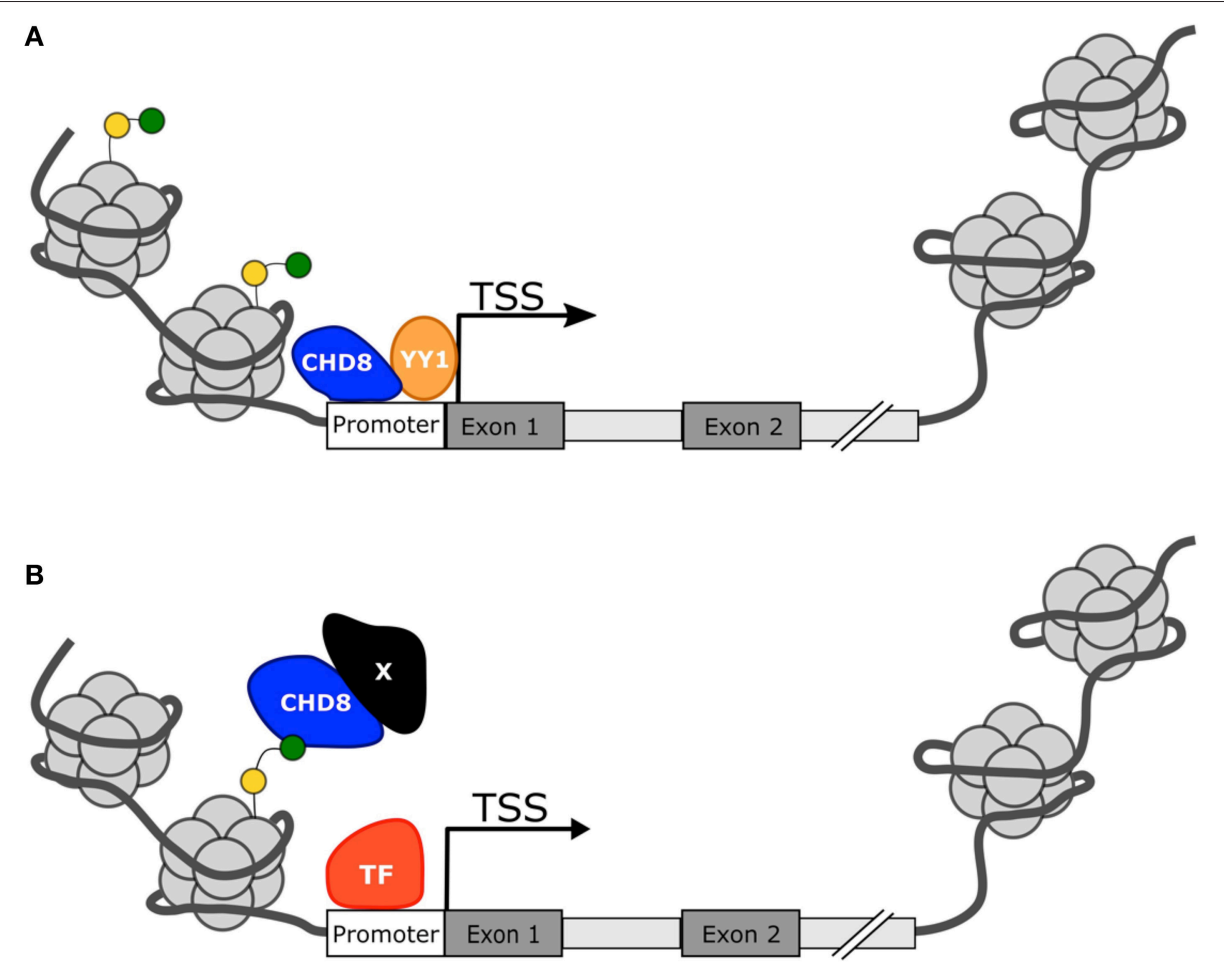

FIGURE 3 | Proposed mechanisms for CHD8 transcriptional activation. (A) CHD8 is most commonly found near active transcription start sites with histone modifications H3K4me3 (green circle) or H3K27ac (yellow circle). CHD8 may directly activate genes by directly binding near the transcriptional start site and promote transcription factor activity or recruitment. (B) CHD8 may indirectly activate genes through interactions between modified histone sites and other co-regulators to make chromatin more assessable.

as co-expression and protein-protein interactions may improve identification of ASD and other neurodevelopmental disorder specific networks, as proposed recently (Hormozdiari et al., 2015). Through the combination of rapidly advancing genetics and the development of relevant neural models, ASD biology is slowly beginning to resolve, and providing the potential for individualized approaches to therapy.

\section{AUTHOR CONTRIBUTIONS}

RAB: writing, editing, and figure conception, MBP: writing and figure design, BJO: writing, editing, and figure conception.

\section{REFERENCES}

Abrahams, B. S., and Geschwind, D. H. (2008). Advances in autism genetics: on the threshold of a new neurobiology. Nat. Rev. Genet. 9, 341-355. doi: $10.1038 / \operatorname{nrg} 2346$

American Psychaitric Association (2013). "Neurodevelopmental disorders," in Diagnostic and Statistical Manual of Mental Disorders, 5th Edn (Washington, DC: American Psychiatric Association). doi: 10.1176/appi.books.9780890425596.dsm01

Basu, S. N., Kollu, R., and Banerjee-Basu, S. (2009). AutDB: a gene reference resource for autism research. Nucleic Acids Res. 37, D832-D836. doi: $10.1093 /$ nar/gkn835

\section{ACKNOWLEDGMENTS}

BJO currently holds and receives support from the Sloan Research Fellowship in Neurosciences (Alfred P. Sloan Foundation), Klingenstein-Simons Fellowship in Neurosciences (Esther A. \& Joseph Klingenstein Fund \& the Simons Foundation), and the NARSAD-Atherton Foundation Young Investigator Award (Brain and Behavior Research Foundation). We would like to thank Dr. Raphael Bernier for his input on the CHD8 mutation phenotype data. We would also like to thank the contributions of families and participants in the described studies. Without them, none of this work would be possible.

Bernier, R., Golzio, C., Xiong, B., Stessman, H. A., Coe, B. P., Penn, O, et al. (2014). Disruptive CHD8 mutations define a subtype of autism early in development. Cell 158, 263-276. doi: 10.1016/j.cell.2014. 06.017

Bernstein, B. E., Stamatoyannopoulos, J. A., Costello, J. F., Ren, B., Milosavljevic, A., Meissner, A., et al. (2010). The NIH roadmap epigenomics mapping consortium. Nat. Biotechnol. 28, 1045-1048. doi: 10.1038/nbt101 $0-1045$

Blumberg, S. J., Bramlett, M. D., Kogan, M. D., Schieve, L. A., Jones, J. R., and $\mathrm{Lu}$, M. C. (2013). Changes in prevalence of parent-reported autism spectrum disorder in school-aged U.S. children: 2007 to 2011-2012. Natl. Health Stat. Rep. 1-11, 1 p following 11. 
Bronicki, L. M., Redin, C., Drunat, S., Piton, A., Lyons, M., Passemard, S., et al. (2015). Ten new cases further delineate the syndromic intellectual disability phenotype caused by mutations in DYRK1A. Eur. J. Hum. Genet. 11, 1482-1487. doi: 10.1038/ejhg.2015.29

Butler, M. G., Dasouki, M. J., Zhou, X. P., Talebizadeh, Z., Brown, M., Takahashi, T. N., et al. (2005). Subset of individuals with autism spectrum disorders and extreme macrocephaly associated with germline PTEN tumour suppressor gene mutations. J. Med. Genet. 42, 318-321. doi: 10.1136/jmg.2004. 024646

Carvill, G. L., Heavin, S. B., Yendle, S. C., McMahon, J. M., O’Roak, B. J., Cook, J., et al. (2013). Targeted resequencing in epileptic encephalopathies identifies de novo mutations in CHD2 and SYNGAP1. Nat. Genet. 45, 825-830. doi: 10.1038/ng.2646

Centers for Disease Control (2014). Prevalence of autism spectrum disorder among children aged 8 years - autism and developmental disabilities monitoring network, 11 sites, United States, 2010. MMWR Surveill. Summ. 63, $1-21$.

Colvert, E., Tick, B., McEwen, F., Stewart, C., Curran, S. R., Woodhouse, E., et al. (2015). Heritability of autism spectrum disorder in a UK population-based twin sample. JAMA Psychiatry 72, 415-423. doi: 10.1001/jamapsychiatry.2014.3028

Cotney, J., Muhle, R. A., Sanders, S. J., Liu, L., Willsey, A. J., Niu, W., et al. (2015). The autism-associated chromatin modifier CHD8 regulates other autism risk genes during human neurodevelopment. Nat. Commun. 6, 6404. doi: $10.1038 /$ ncomms7404

Delmas, V., Stokes, D. G., and Perry, R. P. (1993). A mammalian DNA-binding protein that contains a chromodomain and an SNF2/SWI2-like helicase domain. Proc. Natl. Acad. Sci. U.S.A. 90, 2414-2418.

De Rubeis, S., He, X., Goldberg, A. P., Poultney, C. S., Samocha, K., Cicek, A. E., et al. (2014). Synaptic, transcriptional and chromatin genes disrupted in autism. Nature 515, 209-215. doi: 10.1038/nature13772

Elsabbagh, M., Divan, G., Koh, Y. J., Kim, Y. S., Kauchali, S., Marcín, C., et al. (2012). Global prevalence of autism and other pervasive developmental disorders. Autism Res. 5, 160-179. doi: 10.1002/aur.239

Geschwind, D. H., and State, M. W. (2015). Gene hunting in autism spectrum disorder: on the path to precision medicine. Lancet Neurol. 11, 1109-1120. doi: 10.1016/s1474-4422(15)00044-7

Girard, S. L., Gauthier, J., Noreau, A., Xiong, L., Zhou, S., Jouan, L., et al. (2011). Increased exonic de novo mutation rate in individuals with schizophrenia. Nat. Genet. 43, 860-863. doi: 10.1038/ng.886

He, X., Sanders, S. J., Liu, L., De Rubeis, S., Lim, E. T., Sutcliffe, J. S., et al. (2013). Integrated model of de novo and inherited genetic variants yields greater power to identify risk genes. PLoS Genet. 9:e1003671. doi: 10.1371/journal.pgen.1003671

Helsmoortel, C., Vulto-Van Silfhout, A. T., Coe, B. P., Vandeweyer, G., Rooms, L., Van den Ende, J., et al. (2014). A SWI/SNF-related autism syndrome caused by de novo mutations in ADNP. Nat. Genet. 46, 380-384. doi: 10.1038/ng.2899

Hormozdiari, F., Penn, O., Borenstein, E., and Eichler, E. E. (2015). The discovery of integrated gene networks for autism and related disorders. Genome Res. 25, 142-154. doi: 10.1101/gr.178855.114

Iossifov, I., O’Roak, B. J., Sanders, S. J., Ronemus, M., Krumm, N., Levy, D., et al. (2014). The contribution of de novo coding mutations to autism spectrum disorder. Nature 515, 216-221. doi: 10.1038/nature13908

Iossifov, I., Ronemus, M., Levy, D., Wang, Z., Hakker, I., Rosenbaum, J., et al. (2012). De novo gene disruptions in children on the autistic spectrum. Neuron 74, 285-299. doi: 10.1016/j.neuron.2012.04.009

Ishihara, K., Oshimura, M., and Nakao, M. (2006). CTCF-dependent chromatin insulator is linked to epigenetic remodeling. Mol. Cell 23, 733-742. doi: 10.1016/j.molcel.2006.08.008

Ji, J., Lee, H., Argiropoulos, B., Dorrani, N., Mann, J., Martinez-Agosto, J. A., et al. (2015). DYRK1A haploinsufficiency causes a new recognizable syndrome with microcephaly, intellectual disability, speech impairment, and distinct facies. Eur. J. Hum. Genet. 11, 1473-1481. doi: 10.1038/ejhg.2015.71

Kong, A., Frigge, M. L., Masson, G., Besenbacher, S., Sulem, P., Magnusson, G., et al. (2012). Rate of de novo mutations and the importance of father's age to disease risk. Nature 488, 471-475. doi: 10.1038/nature11396

Kwon, C. H., Zhu, X., Zhang, J., Knoop, L. L., Tharp, R., Smeyne, R. J., et al. (2001). Pten regulates neuronal soma size: a mouse model of Lhermitte-Duclos disease. Nat. Genet. 29, 404-411. doi: 10.1038/ng781
Langfelder, P., and Horvath, S. (2008). WGCNA: an R package for weighted correlation network analysis. BMC Bioinformatics 9:559. doi: 10.1186/14712105-9-559

Lawrence, M. S., Stojanov, P., Mermel, C. H., Robinson, J. T., Garraway, L. A., Golub, T. R., et al. (2014). Discovery and saturation analysis of cancer genes across 21 tumour types. Nature 505, 495-501. doi: 10.1038/nature12912

Liu, L., Lei, J., Sanders, S. J., Willsey, A. J., Kou, Y., Cicek, A. E., et al. (2014). DAWN: a framework to identify autism genes and subnetworks using gene expression and genetics. Mol. Autism 5:22. doi: 10.1186/2040-2 392-5-22

Lord, C., Rutter, M., Dilavore, P., and Risi, S. (1999). Autism Diagnostic Observation Schedule-WPS Edition. Los Angeles, CA: Western Psychological Services.

Lord, C., Rutter, M., and Le Couteur, A. (1994). Autism diagnostic interviewrevised: a revised version of a diagnostic interview for caregivers of individuals with possible pervasive developmental disorders. J. Autism Dev. Disord. 24, 659-685.

Luikart, B. W., Schnell, E., Washburn, E. K., Bensen, A. L., Tovar, K. R., and Westbrook, G. L. (2011). Pten knockdown in vivo increases excitatory drive onto dentate granule cells. J. Neurosci. 31, 4345-4354. doi: 10.1523/jneurosci.0061-11.2011

Marfella, C. G. A., and Imbalzano, A. N. (2007). The Chd family of chromatin remodelers. Mutat. Res. 618, 30-40. doi: 10.1016/j.mrfmmm.2006.07.012

Mccarthy, S. E., Gillis, J., Kramer, M., Lihm, J., Yoon, S., Berstein, Y., et al. (2014). De novo mutations in schizophrenia implicate chromatin remodeling and support a genetic overlap with autism and intellectual disability. Mol. Psychiatry 19, 652-658. doi: 10.1038/mp.2014.29

Neale, B. M., Kou, Y., Liu, L., Ma’ayan, A., Samocha, K. E., Sabo, A., et al. (2012). Patterns and rates of exonic de novo mutations in autism spectrum disorders. Nature 485, 242-245. doi: 10.1038/nature11011

Ng, S. B., Buckingham, K. J., Lee, C., Bigham, A. W., Tabor, H. K., Dent, K. M., et al. (2010). Exome sequencing identifies the cause of a mendelian disorder. Nat. Genet. 42, 30-35. doi: 10.1038/ng.499

Nishiyama, M., Nakayama, K., Tsunematsu, R., Tsukiyama, T., Kikuchi, A., and Nakayama, K. I. (2004). Early embryonic death in mice lacking the beta-catenin-binding protein Duplin. Mol. Cell. Biol. 24, 8386-8394. doi: 10.1128/mcb.24.19.8386-8394.2004

Nishiyama, M., Oshikawa, K., Tsukada, Y., Nakagawa, T., Iemura, S., Natsume, T., et al. (2009). CHD8 suppresses p53-mediated apoptosis through histone H1 recruitment during early embryogenesis. Nat. Cell Biol. 11, 172-182. doi: $10.1038 /$ ncb 1831

O’Roak, B. J., Deriziotis, P., Lee, C., Vives, L., Schwartz, J. J., Girirajan, S., et al. (2011). Exome sequencing in sporadic autism spectrum disorders identifies severe de novo mutations. Nat. Genet. 43, 585-589. doi: 10.1038/ng.835

O’Roak, B. J., Stessman, H. A., Boyle, E. A., Witherspoon, K. T., Martin, B., Lee, C., et al. (2014). Recurrent de novo mutations implicate novel genes underlying simplex autism risk. Nat. Commun. 5, 5595. doi: 10.1038/ncomms6595

O’Roak, B. J., Vives, L., Fu, W., Egertson, J. D., Stanaway, I. B., Phelps, I. G., et al. (2012a). Multiplex targeted sequencing identifies recurrently mutated genes in autism spectrum disorders. Science 338, 1619-1622. doi: $10.1126 /$ science. 1227764

O’Roak, B. J., Vives, L., Girirajan, S., Karakoc, E., Krumm, N., Coe, B. P., et al. (2012b). Sporadic autism exomes reveal a highly interconnected protein network of de novo mutations. Nature 485, 246-250. doi: 10.1038/nature10989

Parikshak, N. N., Luo, R., Zhang, A., Won, H., Lowe, J. K., Chandran, V., et al. (2013). Integrative functional genomic analyses implicate specific molecular pathways and circuits in autism. Cell 155, 1008-1021. doi: 10.1016/j.cell.2013.10.031

Risch, N., Hoffmann, T. J., Anderson, M., Croen, L. A., Grether, J. K., and Windham, G. C. (2014). Familial recurrence of autism spectrum disorder: evaluating genetic and environmental contributions. Am. J. Psychiatry 171, 1206-1213. doi: 10.1176/appi.ajp.2014.13101359

Rodríguez-Paredes, M., Ceballos-Chávez, M., Esteller, M., García-Domínguez, M., and Reyes, J. C. (2009). The chromatin remodeling factor CHD8 interacts with elongating RNA polymerase II and controls expression of the cyclin E2 gene. Nucleic Acids Res. 37, 2449-2460. doi: 10.1093/nar/gkp101

Ronan, J. L., Wu, W., and Crabtree, G. R. (2013). From neural development to cognition: unexpected roles for chromatin. Nat. Rev. Genet. 14, 347-359. doi: $10.1038 / \operatorname{nrg} 3413$ 
Rosenfeld, J. A., Ballif, B. C., Torchia, B. S., Sahoo, T., Ravnan, J. B., Schultz, R., et al. (2010). Copy number variations associated with autism spectrum disorders contribute to a spectrum of neurodevelopmental disorders. Genet. Med. 12, 694-702. doi: 10.1097/GIM.0b013e3181f0c5f3

Sakamoto, I., Kishida, S., Fukui, A., Kishida, M., Yamamoto, H., Hino, S., et al. (2000). A novel beta-catenin-binding protein inhibits beta-catenin-dependent Tcf activation and axis formation. J. Biol. Chem. 275, 32871-32878. doi: 10.1074/jbc.M004089200

Sanders, S. J., Murtha, M. T., Gupta, A. R., Murdoch, J. D., Raubeson, M. J., Willsey, A. J., et al. (2012). De novo mutations revealed by whole-exome sequencing are strongly associated with autism. Nature 485, 237-241. doi: 10.1038/nature10945

Shen, E. H., Overly, C. C., and Jones, A. R. (2012). The Allen Human Brain Atlas: comprehensive gene expression mapping of the human brain. Trends Neurosci. 35, 711-714. doi: 10.1016/j.tins.2012.09.005

Shinawi, M., Liu, P., Kang, S. H., Shen, J., Belmont, J. W., Scott, D. A., et al. (2010). Recurrent reciprocal 16p11.2 rearrangements associated with global developmental delay, behavioural problems, dysmorphism, epilepsy, and abnormal head size. J. Med. Genet. 47, 332-341. doi: 10.1136/jmg.2009.073015

Subtil-Rodríguez, A., Vázquez-Chávez, E., Ceballos-Chavez, M., RodríguezParedes, M., Martín-Subero, J. I., Esteller, M., et al. (2014). The chromatin remodeller CHD8 is required for E2F-dependent transcription activation of S-phase genes. Nucleic Acids Res. 42, 2185-2196. doi: 10.1093/nar/gkt1161

Sugathan, A., Biagioli, M., Golzio, C., Erdin, S., Blumenthal, I., Manavalan, P., et al. (2014). CHD8 regulates neurodevelopmental pathways associated with autism spectrum disorder in neural progenitors. Proc. Natl. Acad. Sci. U.S.A. 111, E4468-E4477. doi: 10.1073/pnas.1405266111

Talkowski, M. E., Rosenfeld, J. A., Blumenthal, I., Pillalamarri, V., Chiang, C., Heilbut, A., et al. (2012). Sequencing chromosomal abnormalities reveals neurodevelopmental loci that confer risk across diagnostic boundaries. Cell 149, 525-537. doi: 10.1016/j.cell.2012.03.028

Veltman, J. A., and Brunner, H. G. (2012). De novo mutations in human genetic disease. Nat. Rev. Genet. 13, 565-575. doi: 10.1038/nrg3241

Vissers, L. E., De Ligt, J., Gilissen, C., Janssen, I., Steehouwer, M., De Vries, P., et al. (2010). A de novo paradigm for mental retardation. Nat. Genet. 42, 1109-1112. doi: $10.1038 /$ ng.712

Voineagu, I., Wang, X., Johnston, P., Lowe, J. K., Tian, Y., Horvath, S., et al. (2011). Transcriptomic analysis of autistic brain reveals convergent molecular pathology. Nature 474, 380-384. doi: 10.1038/nature10110
Wilkinson, B., Grepo, N., Thompson, B. L., Kim, J., Wang, K., Evgrafov, O. V., et al. (2015). The autism-associated gene chromodomain helicase DNA-binding protein 8 (CHD8) regulates noncoding RNAs and autism-related genes. Transl. Psychiatry 5, e568. doi: 10.1038/tp. 2015.62

Willsey, A. J., Sanders, S. J., Li, M., Dong, S., Tebbenkamp, A. T., Muhle, R. A., et al. (2013). Coexpression networks implicate human midfetal deep cortical projection neurons in the pathogenesis of autism. Cell 155, 997-1007. doi: 10.1016/j.cell.2013.10.020

Woodage, T., Basrai, M. A., Baxevanis, A. D., Hieter, P., and Collins, F. S. (1997). Characterization of the CHD family of proteins. Proc. Natl. Acad. Sci. U.S.A. 94, 11472-11477.

Xu, B., Roos, J. L., Dexheimer, P., Boone, B., Plummer, B., Levy, S., et al. (2011). Exome sequencing supports a de novo mutational paradigm for schizophrenia. Nat. Genet. 43, 864-868. doi: 10.1038/ng.902

Yuan, C. C., Zhao, X., Florens, L., Swanson, S. K., Washburn, M. P., and Hernandez, N. (2007). CHD8 associates with human Staf and contributes to efficient U6 RNA polymerase III transcription. Mol. Cell. Biol. 27, 8729-8738. doi: $10.1128 / \mathrm{mcb} .00846-07$

Zahir, F., Firth, H. V., Baross, A., Delaney, A. D., Eydoux, P., Gibson, W. T., et al. (2007). Novel deletions of $14 \mathrm{q} 11.2$ associated with developmental delay, cognitive impairment and similar minor anomalies in three children. J. Med. Genet. 44, 556-561. doi: 10.1136/jmg.2007. 050823

Conflict of Interest Statement: The authors declare that the research was conducted in the absence of any commercial or financial relationships that could be construed as a potential conflict of interest.

Brian J. O'Roak is an inventor on patent PCT/US2009/30620: mutations in contactin associated protein 2 are associated with increased risk for idiopathic autism.

Copyright $\odot 2015$ Barnard, Pomaville and O'Roak. This is an open-access article distributed under the terms of the Creative Commons Attribution License (CC BY). The use, distribution or reproduction in other forums is permitted, provided the original author(s) or licensor are credited and that the original publication in this journal is cited, in accordance with accepted academic practice. No use, distribution or reproduction is permitted which does not comply with these terms. 